\title{
Towards Understanding Mechanisms Governing Cytotoxicity of Metal Oxides Nanoparticles: Hints from Nano-QSAR Studies
}

\author{
A. Gajewicz ${ }^{a}$, N. Schaeublin ${ }^{b}$, \\ B. Rasulev ${ }^{c}$, S. Hussain ${ }^{b}$, D. Leszczynska ${ }^{d}$, T. Puzyn ${ }^{a}$ and \\ J. Leszczynski ${ }^{*}$ \\ ${ }^{a}$ Laboratory of Environmental Chemometrics, Institute for Environmental and Human Health \\ Protection, Faculty of Chemistry, University of Gdańsk, Gdańsk, Poland \\ ${ }^{b}$ Biological Interaction of Nanomaterials, Applied Biotechnology Branch, Human Effectiveness Directorate 711th, \\ Human Performance Wing, Air Force Research Laboratory, Wright Patterson Air Force Base OH, USA \\ ${ }^{c}$ Interdisciplinary Nanotoxicity Center, Department of Chemistry and Biochemistry, Jackson \\ State University, Jackson MS, USA \\ ${ }^{a}$ Interdisciplinary Nanotoxicity Center, Department of Civil and Environmental Engineering \\ Jackson State University, Jackson MS, USA
}

\section{Supplementary material}




\section{Symbols and definitions of all calculated molecular descriptors}

TABLE S1

\begin{tabular}{|c|c|c|}
\hline Symbol & Definition of molecular descriptor & $\begin{array}{l}\text { Included in the } \\
\text { model? }\end{array}$ \\
\hline \multicolumn{3}{|c|}{ QUANTUM - MECHANICAL DESCRIPTORS } \\
\hline$\Delta H_{f}^{c}$ & Standard enthalpy of formation of metal oxide nanocluster & Yes \\
\hline TE & Total energy & No \\
\hline EE & Electronic energy & No \\
\hline Core & Core-core repulsion energy & No \\
\hline SAS & Solvent accessible surface & No \\
\hline HOMO & Energy of the Highest Occupied Molecular Orbital & No \\
\hline LUMO & Energy of the Lowest Unoccupied Molecular Orbital & No \\
\hline$\eta$ & Chemical hardness & No \\
\hline S & Total softness & No \\
\hline$E_{g}$ & HOMO-LUMO energy gap & No \\
\hline$\mu$ & Electronic chemical potential & No \\
\hline $\mathrm{E}_{\mathrm{V}}$ & Valance band & No \\
\hline$\overline{E_{C}}$ & Conduction band & No \\
\hline$x^{c}$ & Mulliken's electronegativity & Yes \\
\hline Hard & Parr and Pople's absolute hardness & No \\
\hline Shift & Schuurmann MO shift alpha & No \\
\hline Ahof & Polarizability derived from the heat of formation & No \\
\hline Ad & Polarizability derived from the dipole moment & No \\
\hline \multicolumn{3}{|c|}{ IMAGE DESCRIPTORS } \\
\hline A & Area & No \\
\hline $\mathrm{V}$ & Volume & No \\
\hline$d_{S}$ & Surface diameter & No \\
\hline$d_{V / m}$ & Volume/mass diameter & No \\
\hline $\mathrm{d}_{\text {Sauter }}$ & Volume/surface diameter & No \\
\hline$A_{R \_x}$ & Aspect ratio $\mathrm{X}$ & No \\
\hline$A_{R \_y}$ & Aspect ratio $Y$ & No \\
\hline$P_{X}$ & Porosity X & No \\
\hline$P_{Y}$ & Porosity Y & No \\
\hline$\Psi$ & Sphericity & No \\
\hline $\mathrm{f}_{\text {circ }}$ & Circularity & No \\
\hline
\end{tabular}




\section{Crystallographic data utilized to construct the metal oxides clusters}

TABLE S2

\begin{tabular}{|c|c|}
\hline $\begin{array}{l}\text { Metal } \\
\text { oxide }\end{array}$ & Reference \\
\hline $\mathrm{Al}_{2} \mathrm{O}_{3}$ & $\begin{array}{l}\text { Kondo, S., Tateishi K., Ishizawa N., Structural Evolution of Corundum at High Temperatures. Japanese Journal of Applied Physics, } \\
\text { 2008. 47: p. 616-619 }\end{array}$ \\
\hline $\mathrm{Bi}_{2} \mathrm{O}_{3}$ & $\begin{array}{l}\text { Cornei, N., Tancret N., Abraham F., Mentré O., New epsilon-Bi2O3 metastable polymorph. Inorganic Chemistry, 2006. 26: p. 4886- } \\
4888 .\end{array}$ \\
\hline $\mathrm{CoO}$ & $\begin{array}{l}\text { Saito, S., Nakahigashi K., Shimomura Y., X-Ray Diffraction Study on CoO. Journal of the Physical Society of Japan, 1966. 21: p. 850- } \\
860 .\end{array}$ \\
\hline $\mathrm{Cr}_{2} \mathrm{O}_{3}$ & $\begin{array}{l}\text { Finger, L.W., Hazen R.M., Crystal structure and isothermal compression of } \mathrm{Fe} 2 \mathrm{O} 3, \mathrm{Cr} 2 \mathrm{O} 3 \text {, and V2O3 to } 50 \text { kbars Journal of Applied } \\
\text { Physics, 1980. 51: p. 5362-5368 }\end{array}$ \\
\hline $\mathrm{Fe}_{2} \mathrm{O}_{3}$ & $\begin{array}{l}\text { Hill, A.H., Jiao F., Bruce P.G., Harrison A., Kockelmann W., Ritter C., Neutron Diffraction Study of Mesoporous and Bulk Hematite, a- } \\
\text { Fe2O3. Chemistry of Materials, 2008. 20: p. 4891-4899. }\end{array}$ \\
\hline $\ln _{2} \mathrm{O}_{3}$ & $\begin{array}{l}\text { Prewitt, C.T., Shannon R.D., Rogers D.B., The C Rare Earth Oxide-Corundum Transition and Crystal Chemistry of Oxides Having the } \\
\text { Corundum Structure. Inorganic Chemistry, 1969. 8: p. 1985-1993. }\end{array}$ \\
\hline $\mathrm{La}_{2} \mathrm{O}_{3}$ & $\begin{array}{l}\text { Wu, B., Zinkevich M., Aldinger F., Wen D., Chen L., Ab initio study on structure and phase transition of A-and B-type rare-earth } \\
\text { sesquioxides Ln2O3 ( } \mathrm{Ln}=\mathrm{La}-\mathrm{Lu}, \mathrm{Y} \text {, and Sc) based on density function theory. Journal of Solid State Chemistry, 2007. 180: p. 3280- } \\
3287 .\end{array}$ \\
\hline $\mathrm{Mn}_{2} \mathrm{O}_{3}$ & $\begin{array}{l}\text { Norrestam, R., Ingri N., Östlund E., Bloom G., Hagen G., alpha-Manganese(III) Oxide --- a C-Type Sesquioxide of Orthorhombic } \\
\text { Symmetry Acta Chemica Scandinavica, 1967. 21: p. 2871-2884. }\end{array}$ \\
\hline NiO & $\begin{array}{l}\text { Shimomura, Y., Kojima M., Saito S., Crystal structure of ferromagnetic nickel oxide. Journal of the Physical Society of Japan, } 1956 . \\
\text { 11: p. 1136-1146. }\end{array}$ \\
\hline $\mathrm{Sb}_{2} \mathrm{O}_{3}$ & $\begin{array}{l}\text { Whitten, A.E., Dittrich B., Spackman M.A., Turner P., Brown T.C., Charge density analysis of two polymorphs of antimony(III) oxide. } \\
\text { Dalton Transactions, 2004. 7: p. 23-29. }\end{array}$ \\
\hline $\mathrm{SiO}_{2}$ & $\begin{array}{l}\text { Martinez, J.R., Palomares-Sanchez S., Ortega-Zarzosa G., Ruiz F., Chumakov Y., Rietveld refinement of amorphous SiO2 prepared } \\
\text { via sol-gel method. Materials Letters, 2006. 60: p. 3526-3529. }\end{array}$ \\
\hline $\mathrm{SnO}_{2}$ & $\begin{array}{l}\text { Gracia, L., Beltrán A., Andrés J., Characterization of the high-pressure structures and phase transformations in SnO2. A density } \\
\text { functional theory study. The Journal of Physical Chemistry B, 2007. 111: p. 6479-6485. }\end{array}$ \\
\hline $\mathrm{TiO}_{2}$ & $\begin{array}{l}\text { Swamy, V., Dubrovinsky L.S., Dubrovinskaia N.A., Langenhorst F., Simionovici A.S., Drakopoulos M., Dmitriev V., Weber H.P., Size } \\
\text { effects on the structure and phase transition behavior of baddeleyite TiO2. Solid State Communications, 2005. 134: p. 541-546. }\end{array}$ \\
\hline $\mathrm{V}_{2} \mathrm{O}_{3}$ & $\begin{array}{l}\text { Rozier, P., Ratuszna A., Galy J., Comparative structural and electrical studies of V2O3 and V2-xNixO3 }(0<x<0.75) \text { solid solution. } \\
\text { Zeitschrift fur Anorganische und Allgemeine Chemie, 2002. 628: p. 1236-1242. }\end{array}$ \\
\hline $\mathrm{WO}_{3}$ & Woodward, P.M., Sleight A.W., Vogt T., Ferroelectric Tungsten Trioxide Journal of Solid State Chemistry, 1997. 131: p. 9-17. \\
\hline $\mathrm{Y}_{2} \mathrm{O}_{3}$ & $\begin{array}{l}\text { Santos, C., Strecker K., Suzuki P.A., Kycia S., Silva O.M.M., Silva C., Stabilization of alpha-SIALONs using a rare-earth mixed oxide } \\
\text { (RE2O3) as sintering additive. Materials Research Bulletin, 2005. 40: p. 1094-1103. }\end{array}$ \\
\hline $\mathrm{ZnO}$ & $\begin{array}{l}\text { Singhal, R.K., Samariya A., Xing Y.-T., Kumar S., Dolia S.N., Deshpande U.P., Shripathi T., Saitovitch E.B., Electronic and magnetic } \\
\text { properties of Co-doped Zn O diluted magnetic semiconductor. The Journal of Alloys and Compounds, 2010. 496: p. 324-330. }\end{array}$ \\
\hline $\mathrm{ZrO}_{2}$ & $\begin{array}{l}\text { Naray-Szabo, S., Zur Struktur des Baddeleyits ZrO2. Zeitschrift fuer Kristallographie, Kristallgeometrie, Kristallphysik, Kristallchemie, } \\
\text { 1936. 94: p. 414-416. }\end{array}$ \\
\hline
\end{tabular}


List of calculated quantum - mechanical descriptors

TABLE S3

\begin{tabular}{|c|c|c|c|c|c|c|c|c|c|c|c|c|c|c|c|c|c|c|c|}
\hline \multirow{2}{*}{$\begin{array}{l}\text { Metal } \\
\text { oxide }\end{array}$} & $\Delta \mathbf{H}_{f}^{\mathrm{c}}$ & TE & EE & Core & SAS & номо & LUMO & $n$ & s & $E_{g}$ & $\mu$ & $\mathrm{E}_{\mathrm{v}}$ & $E_{c}$ & $x^{c}$ & Hard & Shift & Ahof & $\mathrm{Ad}$ & \multirow{2}{*}{$\begin{array}{l}\text { Metal } \\
\text { oxide }\end{array}$} \\
\hline & $\mathrm{kcal} / \mathrm{mol}$ & $\mathrm{eV}$ & $\mathrm{eV}$ & $\mathrm{eV}$ & $A^{\wedge} 2$ & $\mathrm{eV}$ & $\mathrm{eV}$ & $\mathrm{eV}$ & $\mathrm{eV}$ & eV & eV & eV & eV & eV & eV & $\mathrm{eV}$ & $A^{\wedge} 3$ & $A^{\wedge} 3$ & \\
\hline $\mathrm{Al}_{2} \mathrm{O}_{3}$ & -600.0 & -2755.8 & -11997.7 & 9242.0 & 307.16 & -8.53 & 1.66 & -5.09 & -0.10 & -10.18 & -3.44 & 1.66 & -8.53 & 3.44 & 5.09 & -3.44 & 17.83 & 17.79 & $\mathrm{Al}_{2} \mathrm{O}_{3}$ \\
\hline $\mathrm{Bi}_{2} \mathrm{O}_{3}$ & -148.5 & -2864.3 & -11242.7 & 8378.4 & 251.06 & -9.43 & $\begin{array}{l}-1.25 \\
\end{array}$ & -4.09 & -0.12 & \begin{tabular}{|l|}
-8.18 \\
\end{tabular} & -5.34 & -1.25 & $\begin{array}{l}-9.43 \\
\end{array}$ & 5.34 & 4.09 & -5.34 & 19.92 & $\begin{array}{l}19.84 \\
\end{array}$ & $\mathrm{Bi}_{2} \mathrm{O}_{3}$ \\
\hline $\mathrm{CoO}$ & $\begin{array}{l}-786.8 \\
\end{array}$ & -5378.2 & -45466.7 & 40088.5 & 347.56 & -9.21 & -5.67 & -1.77 & -0.28 & -3.55 & $\begin{array}{l}-7.44 \\
\end{array}$ & -5.67 & $\begin{array}{l}-9.21 \\
\end{array}$ & 7.44 & 1.50 & -8.25 & 45.50 & 45.50 & $\mathrm{CoO}$ \\
\hline $\mathrm{Cr}_{2} \mathrm{O}_{3}$ & -235.3 & -2507.8 & -10028.3 & 7520.4 & 167.30 & -8.25 & -0.46 & -3.89 & -0.13 & $\begin{array}{l}-7.79 \\
\end{array}$ & -4.36 & -0.46 & -8.25 & 4.36 & 3.89 & -4.36 & 16.35 & 16.02 & $\mathrm{Cr}_{2} \mathrm{O}_{3}$ \\
\hline $\mathrm{Fe}_{2} \mathrm{O}_{3}$ & $\begin{array}{l}-378.5 \\
\end{array}$ & -3480.9 & -13651.6 & 10170.7 & 172.57 & -8.33 & $\begin{array}{l}-0.09 \\
\end{array}$ & -4.12 & -0.12 & \begin{tabular}{|l|}
-8.24 \\
\end{tabular} & -4.21 & -0.09 & -8.33 & 4.21 & 4.44 & -4.57 & 12.33 & 12.32 & $\mathrm{Fe}_{2} \mathrm{O}_{3}$ \\
\hline $\ln _{2} \mathrm{O}_{3}$ & $\begin{array}{l}-52.1 \\
\end{array}$ & -1961.0 & -6085.1 & 4124.1 & 191.20 & -10.62 & -2.95 & -3.84 & -0.13 & -7.68 & -6.78 & -2.95 & -10.62 & 6.78 & 3.84 & -6.78 & 12.44 & 12.43 & $\ln _{2} \mathrm{O}_{3}$ \\
\hline $\mathrm{La}_{2} \mathrm{O}_{3}$ & $\begin{array}{l}-157.7 \\
\end{array}$ & -2486.1 & -8602.4 & 6116.2 & 232.92 & $\begin{array}{l}-10.91 \\
\end{array}$ & -2.00 & -4.45 & -0.11 & -8.90 & -6.45 & -2.00 & -10.91 & 6.45 & 4.45 & -6.45 & 4.95 & 4.95 & $\mathrm{La}_{2} \mathrm{O}_{3}$ \\
\hline $\mathrm{Mn}_{2} \mathrm{O}_{3}$ & $\begin{array}{l}-96.3 \\
\end{array}$ & -5269.3 & -34774.7 & 29505.4 & 321.38 & $\begin{array}{l}-5.02 \\
\end{array}$ & -4.98 & -0.02 & -27.78 & -0.04 & -5.00 & -4.98 & -5.02 & 5.00 & 0.02 & -5.00 & 734.03 & 40.97 & $\mathrm{Mn}_{2} \mathrm{O}_{3}$ \\
\hline $\mathrm{NiO}$ & 68.0 & -4671.0 & -22764.2 & 18093.2 & 179.35 & $\begin{array}{l}-7.78 \\
\end{array}$ & -1.16 & -3.31 & -0.15 & -6.62 & -4.47 & -1.16 & -7.78 & 4.47 & 3.04 & -4.37 & 21.35 & 21.23 & $\mathrm{NiO}$ \\
\hline $\mathrm{Sb}_{2} \mathrm{O}_{3}$ & -206.7 & -2504.8 & -10756.3 & 8251.5 & 255.24 & $\begin{array}{l}-7.96 \\
\end{array}$ & -0.96 & -3.50 & $\begin{array}{l}-0.14 \\
\end{array}$ & -7.00 & -4.46 & -0.96 & $\begin{array}{l}-7.96 \\
\end{array}$ & 4.46 & 3.50 & -4.46 & 23.21 & 23.12 & $\mathrm{Sb}_{2} \mathrm{O}_{3}$ \\
\hline $\mathrm{SiO}_{2}$ & -618.3 & -2764.2 & -10201.7 & 7437.6 & 262.92 & -7.90 & 0.28 & -4.09 & -0.12 & -8.18 & -3.81 & 0.28 & -7.90 & 3.81 & 4.09 & -3.81 & 31.58 & 31.54 & $\mathrm{SiO}_{2}$ \\
\hline $\mathrm{SnO}_{2}$ & $\begin{array}{l}-266.6 \\
\end{array}$ & -3511.0 & -17713.0 & 14202.0 & 359.32 & -6.97 & $\begin{array}{l}-2.18 \\
\end{array}$ & $\begin{array}{l}-2.40 \\
\end{array}$ & -0.21 & -4.79 & -4.57 & -2.18 & -6.97 & 4.57 & 2.40 & -4.57 & 34.22 & 34.14 & $\mathrm{SnO}_{2}$ \\
\hline$\overline{\mathrm{TiO}_{2}}$ & -1492.0 & -2782.9 & -12685.1 & 9902.2 & 271.58 & -7.08 & $\begin{array}{l}-2.73 \\
\end{array}$ & -2.18 & -0.23 & -4.36 & -4.91 & -2.73 & -7.08 & 4.91 & 2.14 & -4.33 & 49.54 & 24.94 & $\mathrm{TiO}_{2}$ \\
\hline $\mathrm{V}_{2} \mathrm{O}_{3}$ & -139.5 & -2168.1 & -7623.9 & 5455.8 & 206.12 & -5.81 & $\begin{array}{l}-0.66 \\
\end{array}$ & -2.58 & -0.19 & -5.15 & -3.24 & -0.66 & -5.81 & 3.24 & 2.58 & $\begin{array}{l}-3.24 \\
\end{array}$ & 26.36 & 26.22 & $\mathrm{~V}_{2} \mathrm{O}_{3}$ \\
\hline $\mathrm{WO}_{3}$ & $\begin{array}{l}-715.4 \\
\end{array}$ & -4310.9 & -21750.8 & 17439.9 & 302.37 & -6.84 & $\begin{array}{l}-6.61 \\
\end{array}$ & -0.11 & -4.41 & -0.23 & -6.73 & -6.61 & $\begin{array}{l}-6.84 \\
\end{array}$ & 6.73 & 0.11 & -6.73 & 40.16 & 39.79 & $\mathrm{WO}_{3}$ \\
\hline $\mathrm{Y}_{2} \mathrm{O}_{3}$ & -135.3 & -2179.8 & -9171.1 & 6991.3 & 636.97 & -5.19 & -1.51 & -1.84 & -0.27 & -3.68 & -3.35 & -1.51 & -5.19 & 3.35 & 1.84 & -3.35 & 107.00 & 106.98 & $\mathrm{Y}_{2} \mathrm{O}_{3}$ \\
\hline $\mathrm{ZnO}$ & -449.4 & -1320.2 & -3221.7 & 1901.5 & 153.42 & \begin{tabular}{|l|}
-11.36 \\
\end{tabular} & -5.30 & -3.03 & -0.16 & -6.07 & -8.33 & -5.30 & $\begin{array}{l}-11.36 \\
\end{array}$ & 8.33 & 3.03 & -8.33 & 9.09 & 9.07 & $\overline{\mathrm{ZnO}}$ \\
\hline $\mathrm{ZrO}_{2}$ & -638.1 & -1331.3 & -3510.7 & 2179.4 & 178.99 & -10.92 & 1.03 & -5.97 & -0.08 & -11.95 & -4.95 & 1.03 & -10.92 & 4.95 & 5.97 & -4.95 & 10.74 & 10.71 & $\mathrm{ZrO}_{2}$ \\
\hline
\end{tabular}


TABLE S4

List of calculated image descriptors

\begin{tabular}{|c|c|c|c|c|c|c|c|c|c|c|c|c|}
\hline \multirow{3}{*}{ etal oxi } & A & V & $d_{s}=\sqrt{\frac{A}{\pi}}$ & $d_{V / m}=\sqrt[3]{\frac{6 V}{\pi}}$ & $d_{\text {Sauter }}=\frac{\sigma V}{A}$ & $A_{R_{-x}}=\frac{d_{\min -x}}{d_{\max _{-} y}}$ & $A_{R_{-y}}=\frac{d_{\min -y}}{d_{\max -x}}$ & $=\sum_{x=1}^{n}$ & $P_{y}=\sum_{i=1}^{n}\left|y_{i}-y_{j}\right|$ & $\psi=\frac{\pi^{1 / 3} 6 V^{2 / 3}}{A}$ & $f_{\text {circ }}=\frac{4 \pi A}{V^{2}}$ & \\
\hline & Area & Volume & Surface diameter & $\begin{array}{c}\text { Volume/ } \\
\text { mass diameter }\end{array}$ & $\begin{array}{l}\text { Volume/surface } \\
\text { diameter }\end{array}$ & Aspect ratio $X$ & Aspect ratio Y & Porosity X & Porosity Y & Sphericity & Circularity & \\
\hline & {$[\mathrm{px}]$} & {$[\mathrm{px}]$} & {$\left[(p x)^{1 / 2}\right]$} & {$\left[(p x)^{1 / 3}\right]$} & {$[-]$} & {$[-]$} & {$[-]$} & {$[\mathrm{px}]$} & {$[\mathrm{px}]$} & {$\left[(p x)^{-13}\right]$} & {$[1 / \mathrm{px}]$} & \\
\hline $\mathrm{Al}_{2} \mathrm{O}_{3}$ & $1.11 \mathrm{E}+09$ & $1.86 \mathrm{E}+06$ & $1.88 \mathrm{E}+04$ & 153 & $1.011 \mathrm{E}-02$ & 0.568 & 0.072 & $5.15 \mathrm{E}+04$ & $-3.16 \mathrm{E}+05$ & $6.62 E-05$ & $4.00 \mathrm{E}-03$ & $\mathrm{Al}_{2} \mathrm{O}_{3}$ \\
\hline $\mathrm{Bi}_{2} \mathrm{O}_{3}$ & $9.80 \mathrm{E}+08$ & $2.05 E+06$ & $1.77 \mathrm{E}+04$ & 158 & $1.25 \mathrm{E}-02$ & 0.402 & 0.055 & $-2.86 \mathrm{E}+05$ & $-1.80 E+05$ & $7.94 \mathrm{E}-05$ & $2.90 \mathrm{E}-03$ & $\overline{\mathrm{Bi}_{2} \mathrm{O}_{3}}$ \\
\hline $\mathrm{CoO}$ & $1.14 E+09$ & $2.14 \mathrm{E}+06$ & $1.90 \mathrm{E}+04$ & 160 & $1.13 \mathrm{E}-02$ & 0.429 & 0.061 & $2.11 \mathrm{E}+04$ & $-2.42 E+05$ & $7.07 E-05$ & $3.10 \mathrm{E}-03$ & $\mathrm{CoO}_{0}$ \\
\hline $\mathrm{Cr}_{r_{2} \mathrm{O}_{3}}$ & $8.70 \mathrm{E}+08$ & $1.52 E+06$ & $1.67 \mathrm{E}+04$ & 143 & $1.05 E-02$ & 0.263 & 0.060 & $-3.42 E+05$ & $-5.51 E+05$ & 7.34E-05 & $\begin{array}{l}4.70 E-03 \\
\end{array}$ & $\mathrm{Cr}_{2} \mathrm{O}_{3}$ \\
\hline $\mathrm{Fe}_{2} \mathrm{O}_{3}$ & $1.09 \mathrm{E}+09$ & $1.78 \mathrm{E}+06$ & $1.86 \mathrm{E}+04$ & 150 & $9.80 \mathrm{E}-03$ & 0.446 & 0.066 & $-1.18 E+05$ & $-3.18 E+05$ & $6.52 E-05$ & $4.30 \mathrm{E}-03$ & $\mathrm{Fe}_{2} \mathrm{O}_{3}$ \\
\hline $\mathrm{In}_{2} \mathrm{O}_{3}$ & $1.07 \mathrm{E}+09$ & $2.04 E+06$ & $1.85 \mathrm{E}+04$ & 157 & $1.14 \mathrm{E}-02$ & 0.499 & 0.065 & $-1.68 E+04$ & $-2.81 E+05$ & $7.24 \mathrm{E}-05$ & $3.20 E-03$ & $\mathrm{In}_{2} \mathrm{O}_{3}$ \\
\hline $\mathrm{La}_{2} \mathrm{O}_{3}$ & $1.10 \mathrm{E}+09$ & $1.91 \mathrm{E}+06$ & $1.87 \mathrm{E}+04$ & 154 & $1.05 E-02$ & 0.524 & 0.072 & $1.33 \mathrm{E}+04$ & $-2.87 E+05$ & $6.79 E-05$ & $3.80 \mathrm{E}-03$ & $\mathrm{La}_{2} \mathrm{O}_{3}$ \\
\hline $\mathrm{Mn}_{2} \mathrm{O}_{3}$ & $1.09 \mathrm{E}+09$ & $2.04 E+06$ & $1.86 \mathrm{E}+04$ & 157 & $1.12 \mathrm{E}-02$ & 0.479 & 0.064 & $7.57 E+04$ & $-2.46 \mathrm{E}+05$ & $7.12 \mathrm{E}-05$ & $3.30 \mathrm{E}-03$ & $\mathrm{Mn}_{2} \mathrm{O}_{3}$ \\
\hline $\mathrm{NiO}$ & $1.19 \mathrm{E}+09$ & $1.96 \mathrm{E}+06$ & $1.95 \mathrm{E}+04$ & 155 & $9.90 \mathrm{E}-03$ & 0.596 & 0.068 & $6.55 \mathrm{E}+03$ & $-3.11 E+05$ & $6.36 \mathrm{E}-05$ & $3.90 \mathrm{E}-03$ & $\mathrm{NiO}$ \\
\hline $\mathrm{Sb}_{2} \mathrm{O}_{3}$ & $9.60 \mathrm{E}+08$ & $1.80 E+06$ & $1.74 \mathrm{E}+04$ & 151 & $1.13 \mathrm{E}-02$ & 0.241 & 0.055 & $3.49 \mathrm{E}+05$ & $-3.72 E+05$ & $7.48 E-05$ & $3.70 \mathrm{E}-03$ & $\frac{\mathrm{Sb}_{2} \mathrm{O}_{3}}{}$ \\
\hline $\mathrm{SiO}_{2}$ & $9.70 E+08$ & $1.81 \mathrm{E}+06$ & $1.76 \mathrm{E}+04$ & 15 & $1.12 \mathrm{E}-02$ & 0.421 & 0.065 & $1.25 \mathrm{E}+05$ & $-4.13 E+05$ & $7.39 E-05$ & $3.70 E-03$ & $\mathrm{SiO}_{2}$ \\
\hline $\mathrm{SnO}_{2}$ & $9.30 \mathrm{E}+08$ & $1.68 \mathrm{E}+06$ & $1.72 \mathrm{E}+04$ & 148 & $1.09 \mathrm{E}-02$ & 0.408 & 0.078 & $-6.23 E+04$ & $-4.28 \mathrm{E}+05$ & $7.37 \mathrm{E}-05$ & $4.10 \mathrm{E}-03$ & $\mathrm{SnO}_{2}$ \\
\hline $\mathrm{TiO}_{2}$ & $9.20 E+08$ & $1.77 \mathrm{E}+06$ & $1.71 \mathrm{E}+04$ & 150 & $1.16 \mathrm{E}-02$ & 0.408 & 0.078 & $9.79 \mathrm{E}+03$ & $-3.98 E+05$ & $7.70 E-05$ & $3.70 \mathrm{E}-03$ & $\mathrm{TiO}_{2}$ \\
\hline $\mathrm{V}_{2} \mathrm{O}_{3}$ & $1.30 \mathrm{E}+09$ & $2.20 \mathrm{E}+06$ & $2.03 E+04$ & 161 & $1.02 E-02$ & 0.569 & 0.059 & $-5.81 E+03$ & $-2.20 E+05$ & $6.29 E-05$ & $3.40 \mathrm{E}-03$ & $\mathrm{~V}_{2} \mathrm{O}_{3}$ \\
\hline $\mathrm{WO}_{3}$ & $9.80 E+08$ & $1.72 E+06$ & $1.76 E+04$ & 149 & $1.06 \mathrm{E}-02$ & 0.409 & 0.067 & $-1.67 E+05$ & $-4.30 E+05$ & $7.111-05$ & $4.10 \mathrm{E}-03$ & $\mathrm{WO}_{3}$ \\
\hline$\frac{\mathrm{Y}_{2} \mathrm{O}_{3}}{n_{3}}$ & $1.28 \mathrm{E}+09$ & $2.26 \mathrm{E}+06$ & $2.02 E+04$ & 163 & $1.06 \mathrm{E}-02$ & 0.633 & 0.058 & $1.02 \mathrm{E}+04$ & $-1.89 E+05$ & $6.51 \mathrm{E}-05$ & $3.10 \mathrm{E}-03$ & $\mathrm{Y}_{2} \mathrm{O}_{3}$ \\
\hline $\mathrm{ZnO}$ & $1.11 \mathrm{E}+09$ & $1.95 E+06$ & $1.88 \mathrm{E}+04$ & 155 & $1.05 E-02$ & 0.535 & 0.071 & $1.83 \mathrm{E}+03$ & $-3.04 E+05$ & $6.80 \mathrm{E}-05$ & $3.70 \mathrm{E}-03$ & $\mathrm{ZnO}$ \\
\hline $\mathrm{ZrO}_{2}$ & $1.20 \mathrm{E}+09$ & $2.22 \mathrm{E}+06$ & $1.95 \mathrm{E}+04$ & 162 & $1.11 \mathrm{E}-02$ & 0.508 & 0.059 & $9.68 \mathrm{E}+03$ & $-2.19 E+05$ & $6.86 \mathrm{E}-05$ & $3.10 \mathrm{E}-03$ & $\mathrm{ZrO}_{2}$ \\
\hline
\end{tabular}

[px] - number of pixels

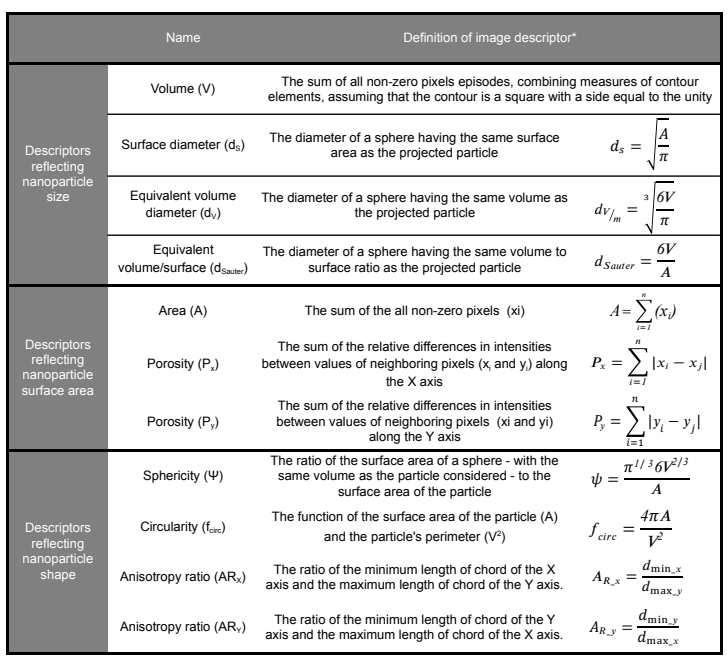

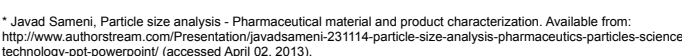


Statistics for the model's coefficients

TABLE S5

\begin{tabular}{|c|c|c|c|c|c|}
\cline { 3 - 6 } \multicolumn{1}{c|}{} & $\mathbf{b}_{\mathrm{i}}$ & std. error & t-value & p-value \\
\hline $\mathbf{b}_{0}$ & intercept & 2.47 & \pm 0.05 & 54.19 & $1.9 \times 10^{-10}$ \\
\hline $\mathbf{b}_{1}$ & coefficient & 0.24 & \pm 0.05 & 5.08 & $1.4 \times 10^{-3}$ \\
\hline $\mathbf{b}_{2}$ & coefficient & 0.39 & \pm 0.05 & 8.21 & $7.7 \times 10^{-5}$ \\
\hline
\end{tabular}




\section{Results for internal validation with leave-one-out algorithm}

TABLE S6

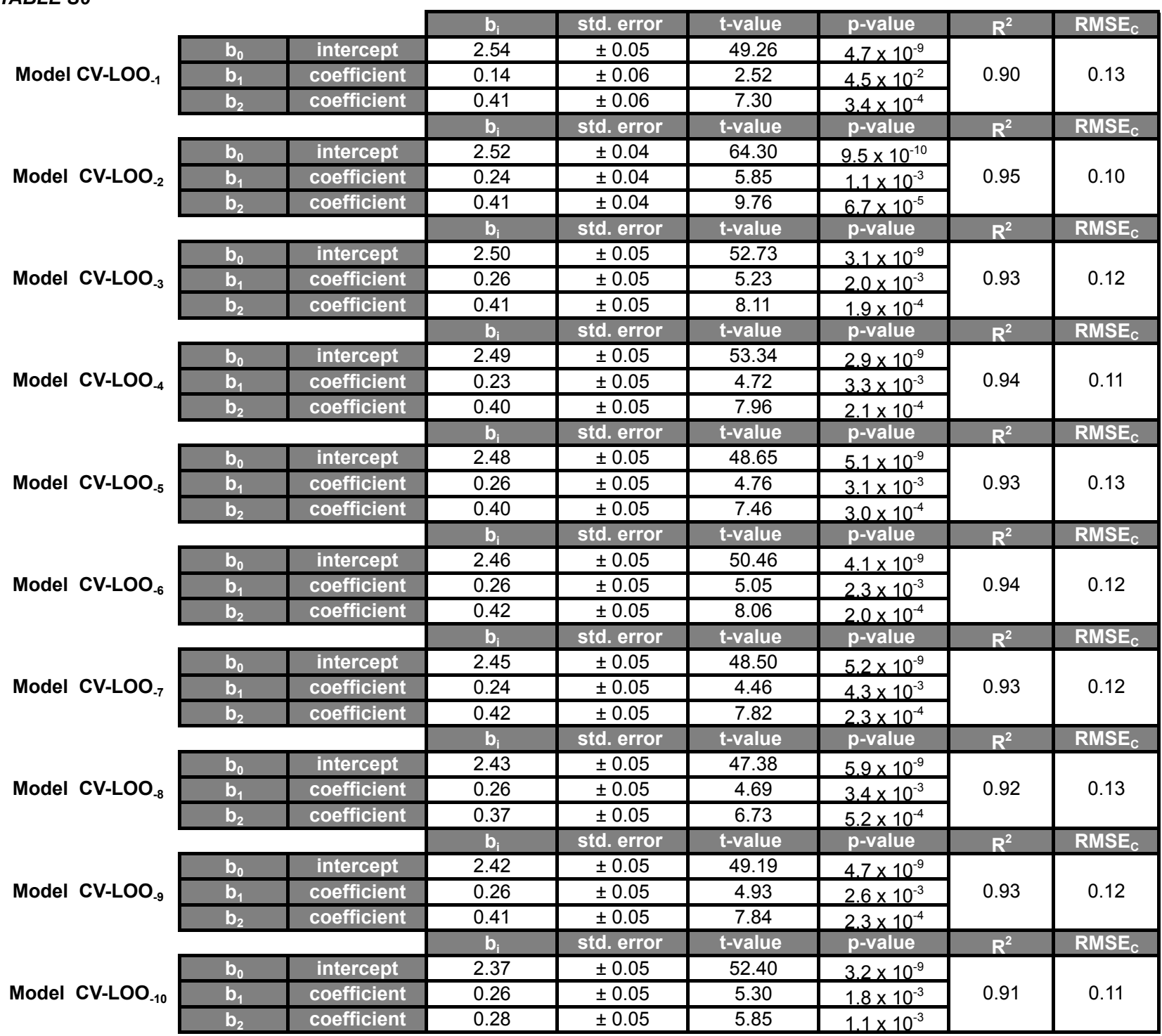


The results of the Y-scrambling test

FIGURE S1

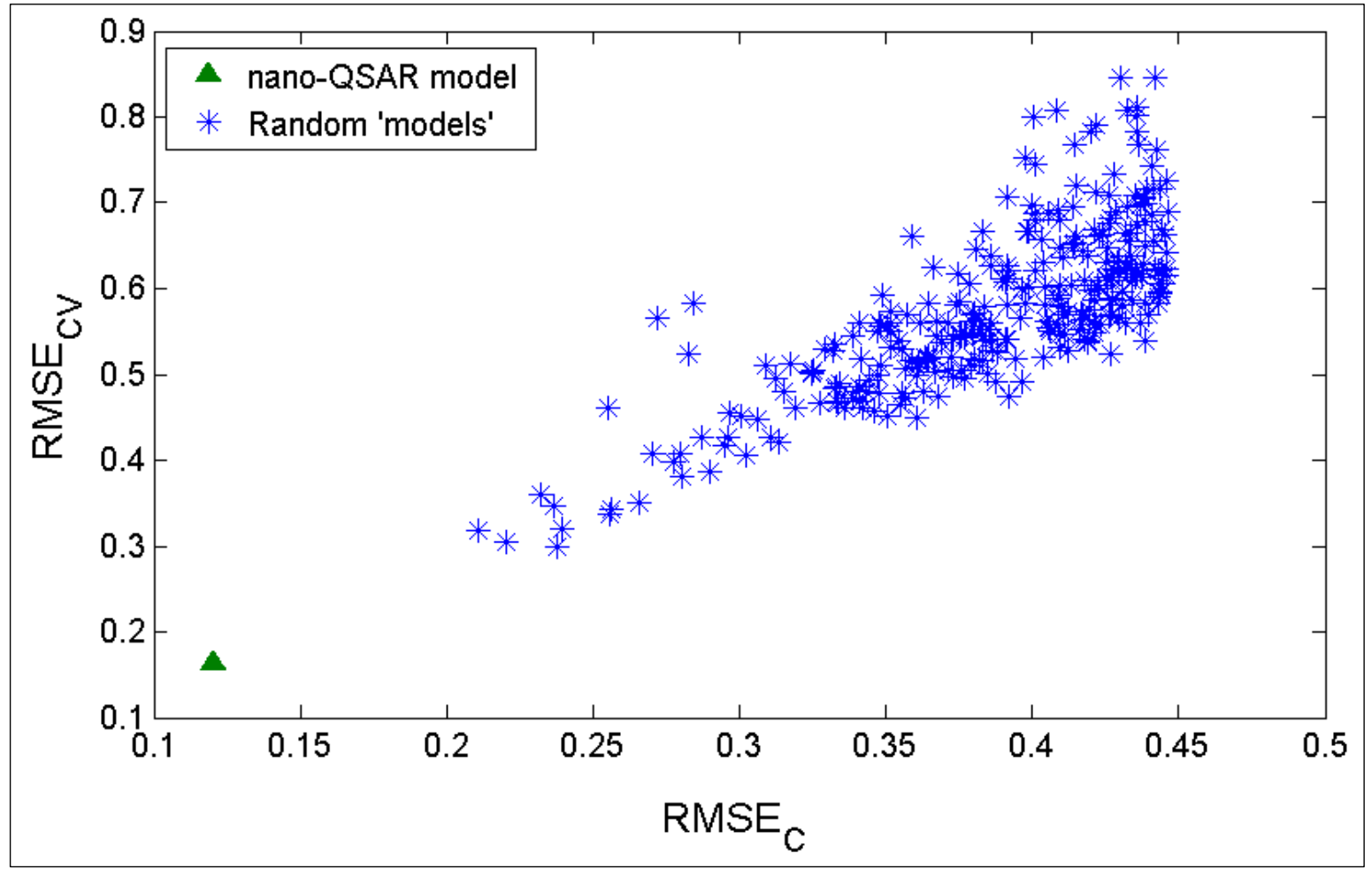


Insubria plot

FIGURE S2

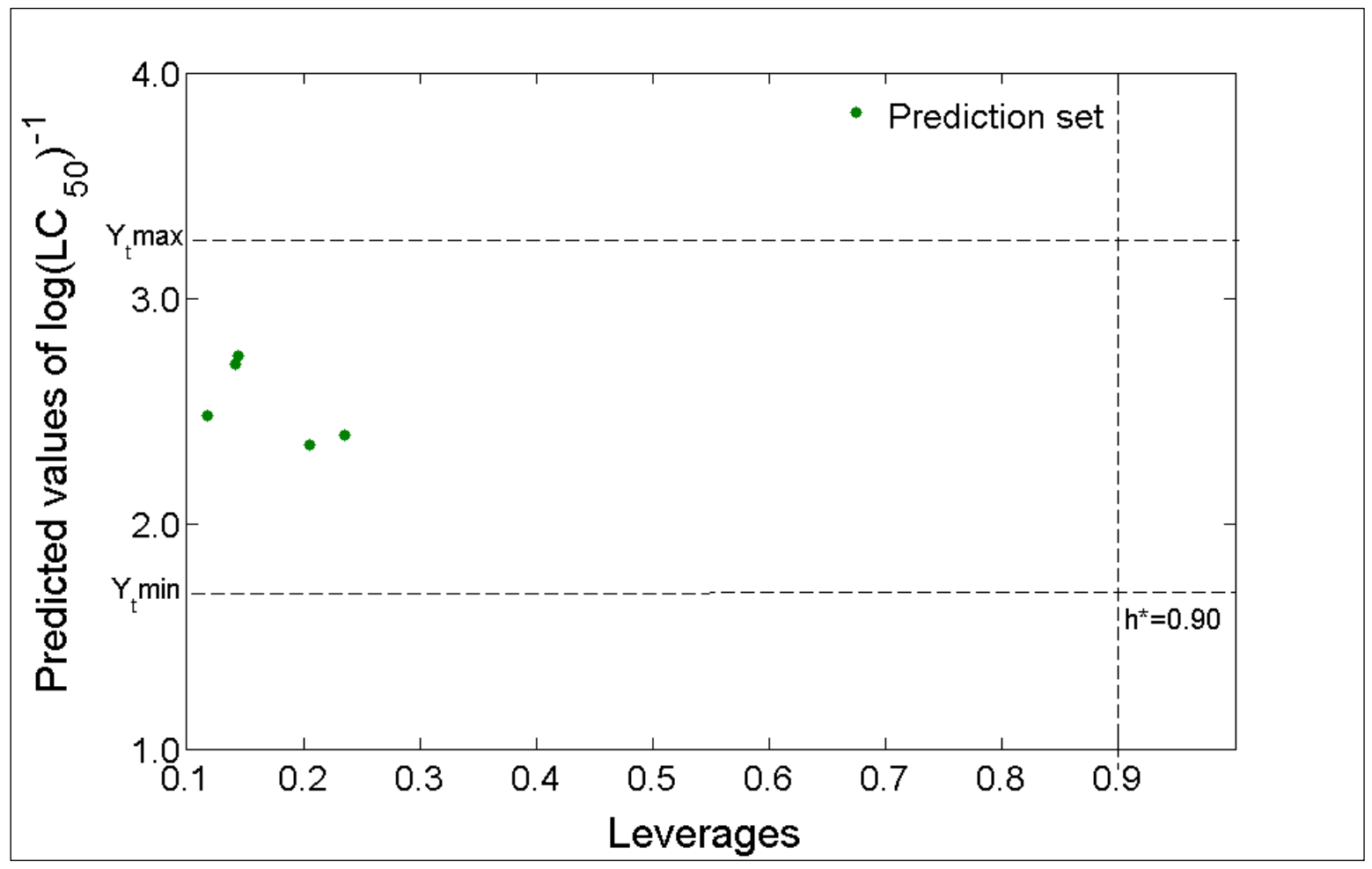

